\title{
Decoding covert somatosensory attention by a BCI system calibrated with tactile sensation
}

\author{
Lin Yao, Xinjun Sheng, Natalie Mrachacz-Kersting, \\ Xiangyang Zhu, Dario Farina, Ning Jiang*
}

\begin{abstract}
Objective: We propose a novel calibration strategy to facilitate the decoding of covert somatosensory attention by exploring the oscillatory dynamics induced by tactile sensation. Methods: It was hypothesized that the similarity of the oscillatory pattern between stimulation sensation (SS, real sensation) and somatosensory attentional orientation (SAO) provides a way to decode covert somatic attention. Subjects were instructed to sense the tactile stimulation, which was applied to the left (SS-L) or the right (SS-R) wrist. The BCI system was calibrated with the sensation data and then applied for online SAO decoding. Results: Both SS and SAO showed oscillatory activation concentrated on the contralateral somatosensory hemisphere. Offline analysis showed that the proposed calibration method led to greater accuracy than the traditional calibration method based on SAO only. This is confirmed by online experiments, where the online accuracy on 15 subjects was $78.8 \pm 13.1 \%$, with 12 subjects $>70 \%$ and 4 subject $>90 \%$. Conclusion: By integrating the stimulus-induced oscillatory dynamics from sensory cortex, covert somatosensory attention can be reliably decoded by a BCI system calibrated with tactile sensation. Significance: Indeed, real tactile sensation is more consistent during calibration than SAO. This brain-computer interfacing approach may find application for stroke and completely locked-in patients with preserved somatic sensation.
\end{abstract}

\section{INTRODUCTION}

A brain-computer interface (BCI) provides a non-muscular communication and control channel between brain activities and the external environment [1], opening a way of interaction for locked-in patients [2]-[4]. By mentally imagining limb movements (e.g., left or right hand) [5], [6], the covert motor intentions can be decoded from brain signals induced by motor imagery (MI) [7], [8]. MI based independent BCIs have received extensive interest [9]-[12] since they do not require external stimuli, contrary to systems based on stimuli such as P300 and Steady-state visual evoked potentials (SSVEP) [13], [14]. Complementary to MI-based BCIs, we recently proposed a new BCI paradigm based on covert sensation attention, and we demonstrated that imagination of somatosensory stimulation can also be decoded from EEG, which we termed somatosensory attentional orientation (SAO) [15]. In this sensory imagery paradigm, subjects shift and maintain their somatosensory attention on parts of their bodies as if the stimulus was applied [15]-[19].

Both MI and SAO are covert mental processes, which are

Lin Yao and Ning Jiang are with Department of Systems Design Engineering, Faculty of Engineering, University of Waterloo, Waterloo, Canada (Corresponding E-mail: ning.jiang@uwaterloo.ca).

Xinjun Sheng and Xiangyang Zhu are with State Key Lab of Mechanical System and Vibration, Shanghai Jiao Tong University, Shanghai, China.

Natalie Mrachacz-Kersting is with Center for Sensory-Motor Interaction, the Faculty of Medicine, Aalborg University, Aalborg, Denmark.

Dario Farina is with Department of Bioengineering, Imperial College London, London, UK. inherently subjective and cannot be directly measured or observed. Because there is no direct measure to ensure that the subject performs the mental task properly during training, it is difficult to ensure a good quality of the training set of data, which is fundamental for accurate BCI control. This problem is especially relevant in patients in the locked-in state with whom the communication is difficult [20]-[23]. The discrepancy between the instructed tasks and the tasks that the patient actually performs during training is one of the limiting factors of BCI systems. Efficient and observable or controllable calibration methods would provide a way to overcome this issue. For example, based on the similarity of the EEG pattern among active movement, passive movement, functional electrical stimulation and MI [24]-[26], a variety of calibration strategies have been developed for MI-based BCI systems. It has been shown that robot-assisted passive movement provides a way for BCI system calibration, so that BCI-driven stroke neurorehabilitation could start immediately after the passive physiotherapy session [27]. This idea was also supported by a clinical study that showed that MI can be well detected by including calibration data from passive movements [28]. Moreover, brain activation patterns induced by neuromuscular electrical stimulation (NMES) can also be used in MI-based BCI training in specific users [29]. For example, we showed that induced sensation with kinesthesia illusion by tendon vibration generates EEG signals that can be used for calibration of MI based BCI [30].

Brain oscillatory dynamics, as quantified by event related desynchronization/synchronization (ERD/ERS) [31], [32], are not only correlated with real or imagined movement, but also with external sensory stimulation processing [33], [34]. We have previously shown that tactile-induced oscillatory dynamics provide a reliable brain signal modality for a tactile BCI [35]-[37]. Furthermore, this oscillatory activity is not only correlated with real tactile sensation, but also with imagined sensation [15]. Therefore, covert somatosensory attention can be used for a new stimulus-independent BCI. However, as SAO is a purely covert mental process, similar to MI, it is difficult to ensure that the subject performs the desired task during training. Therefore, in this study we hypothesized that the stimulus-induced brain oscillatory activation from the somatosensory cortex can be utilized to calibrate SAO-based BCI systems. This hypothesis was evaluated through online BCI experiments.

\section{Methodology}

A. Subjects

Fifteen healthy BCI naïve subjects participated in the experiments (eight female, seven male, all right handed, 
average age $21.5 \pm 1.5$ years). The study was approved by the Ethics Committee of the University of Waterloo, Waterloo, Canada. All participants signed an informed consent form before participation.

All participants finished tactile sensation stimulation (SS) runs and SAO runs as described in the below experiment protocol. Furthermore, 10 subjects (S1-S10) performed two additional runs of SAO tasks before the SS runs. Other 5 subjects performed only SS runs and followed by SAO runs (C1-C5).

\section{B. EEG Recording and Somatosensory Stimulation}

EEG signals were recorded using a 32 channel wireless g.Nautilus EEG system (g.tec, Austria). The electrodes were placed according to the extended 10/20 system. The reference electrode was located on the right earlobe, and the ground electrode on the forehead. A hardware notch filter at $60 \mathrm{~Hz}$ was applied to the raw signals. The signals were digitally sampled at $250 \mathrm{~Hz}$.

Mechanical stimulation was applied to the wrists. Linear resonant actuators $(10 \mathrm{~mm}, \mathrm{C} 10-100$, Precision Microdrives Ltd., typical normalized amplitude $1.4 \mathrm{G}$ ) were used for producing vibrotactile stimulation. The stimulation device produced $27 \mathrm{~Hz}$ sine wave for the left or right wrist. The stimuli were modulated with a $175 \mathrm{~Hz}$ sine carrier wave. These stimuli activate the Pacinian and Meissner corpuscles [38], which are sensitive to frequencies above $100 \mathrm{~Hz}$ and $20-$ $50 \mathrm{~Hz}$, respectively. The amplitude of the vibration was individually adjusted to be between the maximum amplitude $(11.3 \mathrm{um})$ and half of the maximum amplitude at the resonant frequency. The selection of the optimal amplitude was based on individual feedback from the subject, such that they were comfortable with perceiving the vibration.

Before experiment, subjects were tactile stimulated to feel the tactile sensation, and were explicitly instructed not to move their hand or contract the muscle both during real or imagined sensation tasks. Due to the limitation of the g.Nautilus EEG system, no EMG signals were explicitly recorded, as it has been validated that EMG recording was not necessary during the SAO protocol [15].

\section{Experimental Protocol}

During the SS task, subjects were instructed to focus on sensation when the left or right wrist received the tactile stimuli. SS-L (only left hand was stimulated) and SS-R (only right hand was stimulated) were performed in the first two runs. During the SAO task, the subjects were instructed to shift and maintain the somatosensory attention on the left or right hand, and to imagine sensation even when there were no tactile stimuli. SAO-L and SAO-R were performed in the next three runs.

Pre-calibration SAO phase: The experimental paradigm is illustrated in Fig. 1 (A). The subject was seated on a comfortable armchair, with both forearms and hands resting on the armrests. The subjects were instructed to limit their eye, facial and arm movements. A total of 80 trials (40 trials for each task) were performed by the subjects in two runs ( 40
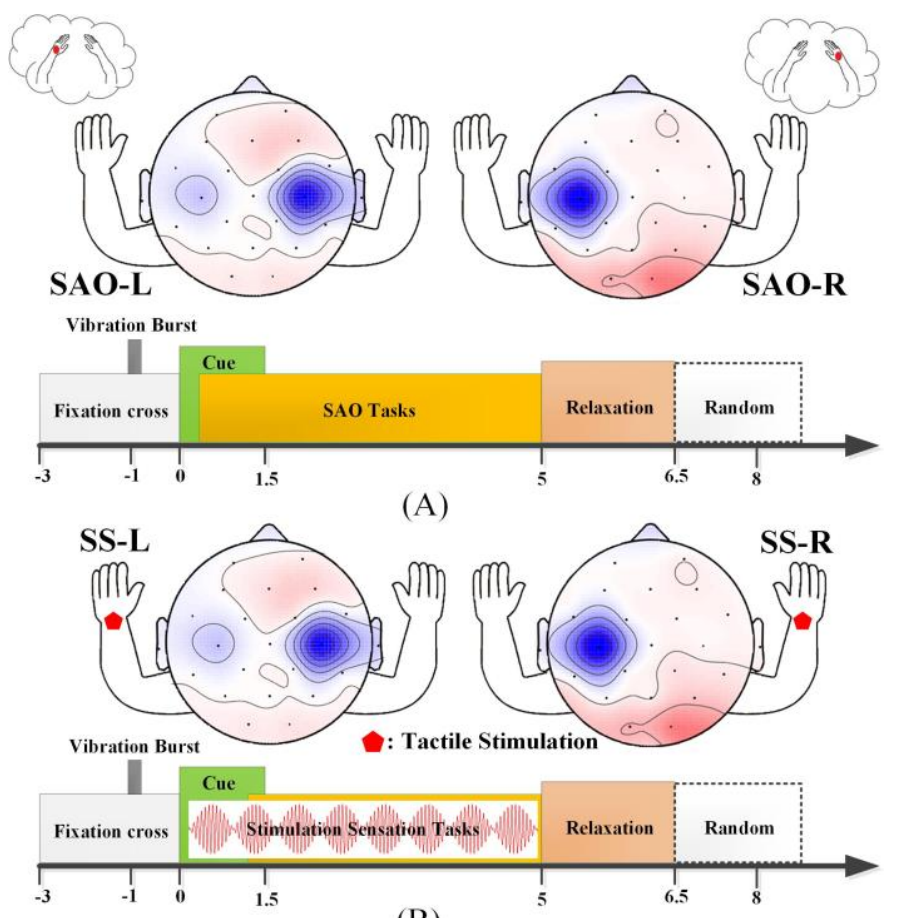

(B)

Figure 1. Illustration of the experiment protocol. (A) Graphic illustration of the experiment protocol of the SI run and the temporal sequence of each trial in this run. Subjects were instructed to shift and maintain the somatosensory attention on left or right hand, while no tactile stimuli was applied (SAO-L or SAO-R Task). (B) Graphic illustration of the experiment protocol of the SS run and the temporal sequence of each trial in this run. Red pentagons indicates the applied unilateral vibration stimulus (on either left or right hand), and the corresponding scalp map of the EEG dynamics (illustration only). Subjects were instructed to focus on sensation on left or right hand when the stimulus was applied to either left or right (SS-L or SS-R Task).

trials per run). There were 2-4 min breaks between the two consecutive runs. The experimental protocol of this run consisted of a sequence of trials. At the beginning of each trial $(\mathrm{T}=-3 \mathrm{~s})$, a visual cue was presented to the subjects on a computer screen located at a distance of $1 \mathrm{~m}$ from the subject. At the beginning of each trial, a white fixation symbol ("+") appeared in the center of the screen. At $\mathrm{T}=-1 \mathrm{~s}$, a vibration pulse stimulated both hands for $200 \mathrm{~ms}$ with the same intensity, to alert the user of the impending task. At $\mathrm{T}=0 \mathrm{~s}$, a red cue pointing either left or right was randomly presented on the computer monitor: 1) a left-pointing arrow instructing the subjects to perform SAO-L task; 2) a right-pointing arrow instructing the subjects to perform SAO-R task. This visual cue (left or right arrow) was superimposed on the fixation symbol and lasted for $1.5 \mathrm{~s}$. The imagined sensation task continued for $5 \mathrm{~s}$, until the fixation symbol disappeared $(\mathrm{T}=5$ s). During the first run, there was no feedback whereas in the subsequent run at the $\mathrm{T}=5 \mathrm{~s}$, there was vibration feedback after the SAO task. The feedback stimulus was applied according to the decoded task type (left or right) for $500 \mathrm{~ms}$. Next there was a relaxation period lasting $1.5 \mathrm{~s}$. Finally, a random time interval of 0 to $2 \mathrm{~s}$ followed the relaxation period before the next trial began.

SS calibration phase: The experimental paradigm is illustrated in Fig. 1 (B). The trial structure was the same as in 
the previous pre-calibration $\mathrm{SAO}$ phase. At $\mathrm{T}=0 \mathrm{~s}$, a red cue pointing either left or right was randomly presented on the computer monitor: 1) a left-pointing arrow instructing the subjects to perform SS-L task; and 2) a right-pointing arrow instructing the subjects to perform SS-R task. This visual cue (left or right arrow) was superimposed on the fixation symbol and lasted for $1.5 \mathrm{~s}$. The subjects were instructed to focus on either the left or right hand where the tactile sensation was applied to as soon as the cue appeared. The sensation task continued for $5 \mathrm{~s}$, until the fixation symbol disappeared ( $\mathrm{T}=5$ s). Next there was a relaxation period lasting $1.5 \mathrm{~s}$. Finally, a random time interval of 0 to $2 \mathrm{~s}$ followed the relaxation period before the next trial began, to prevent subject adaptation. A total of 80 trials ( 40 trials for each task) were performed by the subjects in 2 runs (40 trials per run). There were 2-4 min breaks between the two consecutive runs.

Post-calibration SAO phase: The trial structure was the same as in the previous phase, as shown in Fig. 1 (A). In this part of the experiment, the subjects were required to perform the SAO tasks: 1) a left-pointing arrow corresponded to the SAO-L task; and 2) a right-pointing arrow to the SAO-R task. At $\mathrm{T}=5 \mathrm{~s}$, vibration feedback was provided after the $\mathrm{SAO}$ task. A total of 120 trials (60 trials for each task) were performed by the subjects in 3 runs. There were 2-4 min breaks between consecutive runs.

\section{EEG Dynamics and Time Frequency Decomposition}

Event related desynchronization (ERD) and event related synchronization (ERS) are defined as the percentage of power decrease (ERD) and power increase (ERS) in a defined frequency band with respect to a reference interval (usually taken at a time interval prior to a motor or sensory event) [32]. The frequency band alpha-beta of [8 26] $\mathrm{Hz}$ was adopted in this study for EEG filtering before the ERD/ERS calculation. The reference interval for the ERD/ERS calculation was between $\mathrm{T}=-2 \mathrm{~s}$ and $\mathrm{T}=-1.2 \mathrm{~s}$ prior to the appearance of the cue. The grand averaged ERD/ERS curves from all subjects of the same task were used to determine the activation and deactivation of the cortical areas involved in the mental tasks.

The EEG data was manually corrected for artifacts using the EEGLAB toolbox [39]. Trials contaminated with swallowing and physical movement artifacts (either in baseline or task interval time periods) were excluded from the analysis. Timefrequency decomposition of each trial along each EEG channel was performed to construct the spatio-spectraltemporal structure according to the pre-defined mental tasks. It was calculated every $200 \mathrm{~ms}$ with a hanning tapper, convoluted with a modified sinusoid basis, in which the number of cycles linearly changed with frequency to achieve proper time and frequency resolution [40]. The $\mathrm{R}^{2}$ index (squared Pearson-correlation coefficient between feature and class label) [41], [42] was calculated based on the above spatio-spectral-temporal structures between different mental tasks, and used to locate the component of different EEG channels for the classification of the corresponding mental tasks. The Discriminative Brain Pattern (DBP) was defined as a topographic plot of the $\mathrm{R}^{2}$ index, which was averaged along the task time interval mentioned above, and the frequency bands alpha $(8-13 \mathrm{~Hz})$, beta $(13-26 \mathrm{~Hz})$, or alpha-beta $(8-26$ $\mathrm{Hz}$ ).

\section{E. Algorithms and Performance Evaluation}

Spatial filtering was adopted to reduce the number of channels and to enhance the feature discrimination among the investigated tasks. The spatial filters were determined with the Common Spatial Pattern (CSP) procedure, which has been extensively validated for BCIs [43], [44]. The log-variance of the first three and last three components produced by CSP were chosen as feature vectors, and linear discriminative analysis (LDA) was used for classification.

EEG signals were segmented from $T=1 \mathrm{~s}$ to $\mathrm{T}=4 \mathrm{~s}$ after the appearance of the cue for the analysis. A fourth-order Butterworth filter of [8 26] Hz was applied to the raw EEG signals before the CSP spatial filtering. The trials in the first two runs (SS-L and SS-R tasks) in the SS calibration phase were used to calibrate the BCI system and the parameters were then fixed for subsequent classification of data from the SAO phases. Moreover, for comparison with the conventional calibration method, the pre-calibration SAO data were used to train the BCI system and to classify the post-calibration SAO data.

\section{RESULTS}

\section{A. Neurophysiological Correlation between Real and Imagined Sensation}

The neurophysiological correlation between real and imagined sensation justifies the proposed calibration strategy for covert somatosensory attention decoding. Fig. 2 shows the grand-averaged oscillatory dynamics in both real tactile sensation and imagined sensation. At $\mathrm{T}=-1 \mathrm{~s}$, a vibration burst
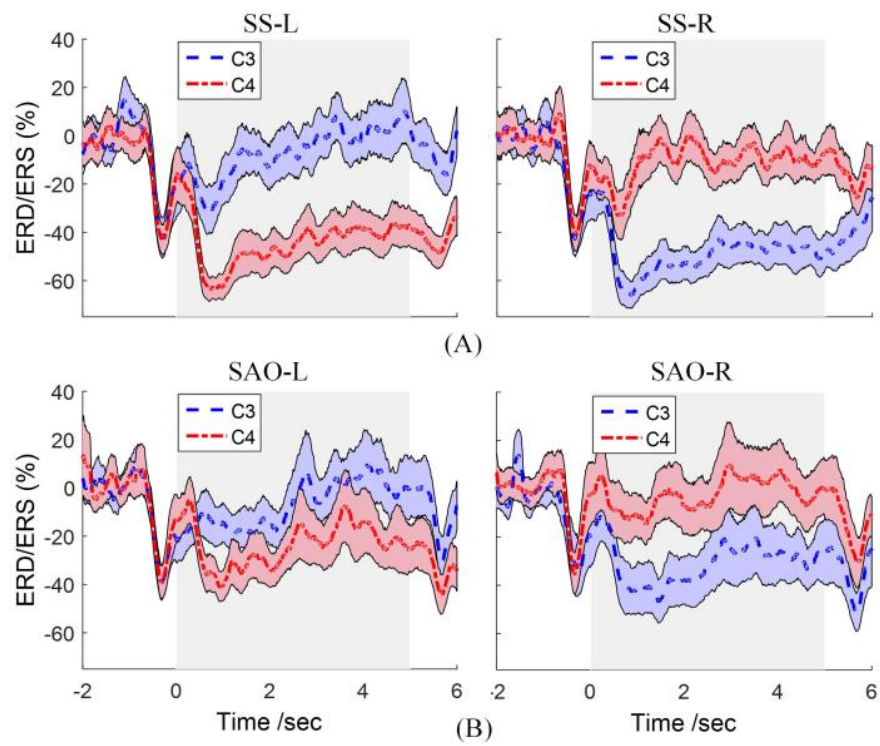

Figure 2. The time varying grand-averaged ERD/ERS curves at smallLaplace filtered $\mathrm{C} 3$ and $\mathrm{C} 4$ channels within the alpha-beta frequency band [8 26] Hz. (A) ERD/ERS corresponding to Stimulation Sensation, where left corresponds to SS-L and right corresponds to SS-R. (B) ERD/ERS corresponding to the SAO task, where left corresponds to SAO-L and right corresponds to SAO-R. Time Os corresponds to the time when the cue appeared ( $3 \mathrm{rd}$ second from the beginning of the trial). 


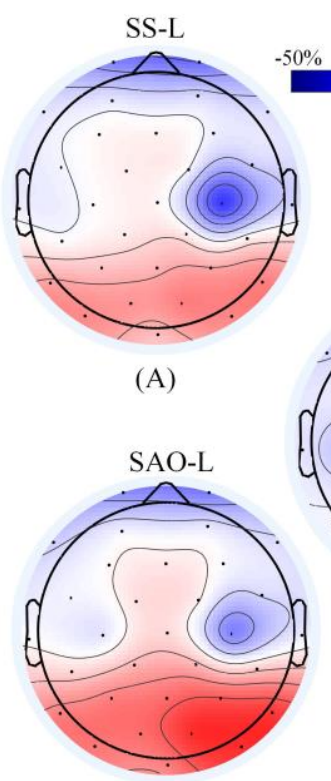

(C)

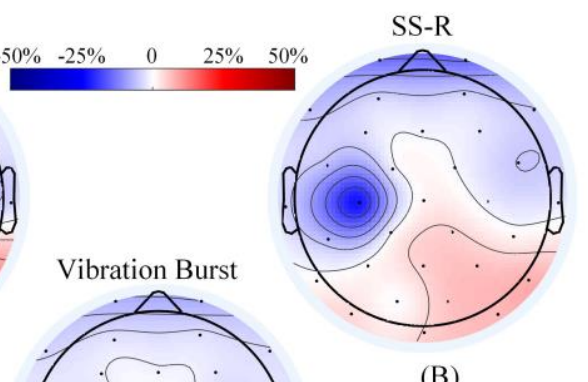

(B)

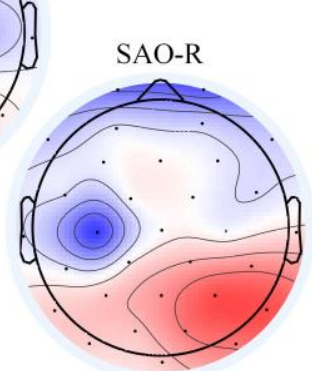

(D)
Figure 3. Grand-averaged ERD/ERS distribution within alpha-beta frequency band [8 26] Hz. (A) ERD/ERS activation with respect to SS-L task. (B) ERD/ERS activation with respect to SS-R task. (3) ERD/ERS activation with respect to SAO-L task. (4) ERD/ERS activation with respect to SAO-R task. (5) ERD/ERS activation with respect to vibration burst ( 1 second before the appearance of the cue). Color bar indicates the ERD/ERS value. Note: ERD/ERS value is averaged between 1 to 4 second after the appearance of the cue in subfigure (A) (B) (C) (D); ERD/ERS value is averaged between $-\mathbf{0 . 5}$ to 0 second before the appearance of the cue in $(\mathbf{E})$.

of $200 \mathrm{~ms}$ was applied to both wrists to alert the subjects to get ready for the task. This corresponded to a clear simultaneous alpha-beta frequency power reduction for both the left hemisphere (C3 channel) and the right hemisphere (C4 channel), with the same strength for all tasks. The real tactile stimulation sensation resulted in a clear difference in the activation pattern between the left and right somatosensory cortex when stimulating either of the two sides. During SS-L sensation, the ERD in the contralateral right hemisphere was much stronger than that in the ipsilateral left hemisphere, and vice versa for SS-R. In contrast, for SAO tasks, these activation dynamics were similar to that in the SS case, i.e., contralateral activation was also observed. Fig. 3 shows the

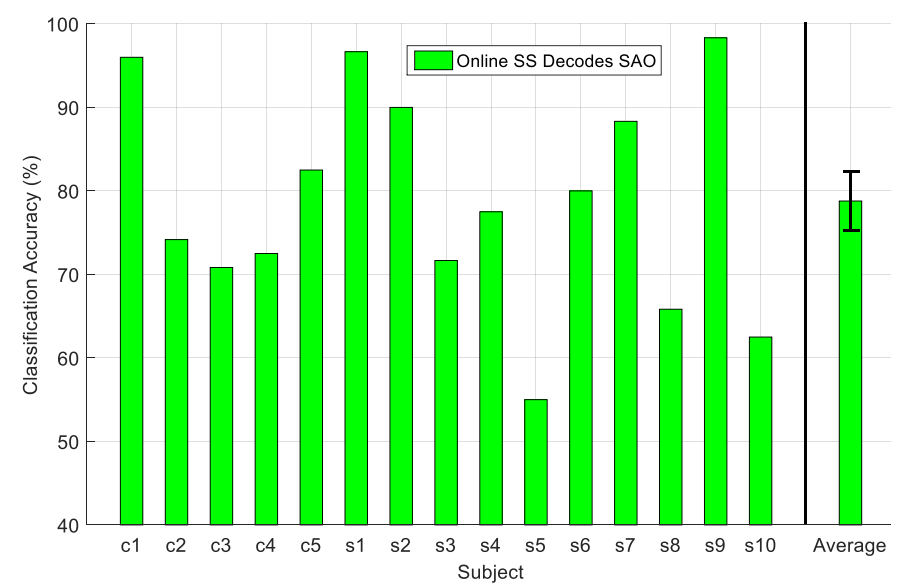

Figure 4. Real-time SAO BCI performance with tactile stimulation sensation for calibration.

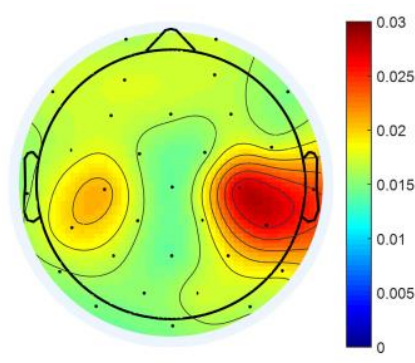

(A)

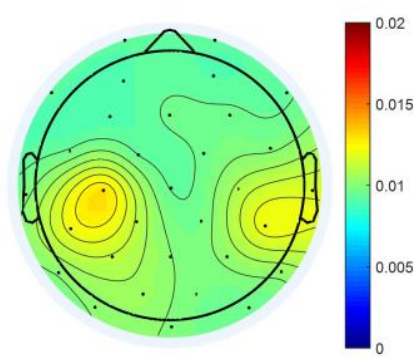

(B)
Figure 5. Grand-averaged $R^{2}$ value distribution from SS tasks and SAO tasks within $\left[8\right.$ 26] $\mathrm{Hz}$ frequency band. (A) $R^{2}$ discriminative information distribution between SS-L and SS-R. (B) $R^{2}$ discriminative information distribution between SAO-L and SAO-R. Note $R^{2}$ was averaged along the temporal dimension corresponding to $T=\left[\begin{array}{ll}1 & 4\end{array}\right] \mathrm{s}$. Note the scale range for SS and SAO was different.

grand-averaged ERD/ERS spatial distribution during different tasks. The vibration burst resulted in both left and right somatosensory cortex co-activation, that was concentrated over the left (C3) and right (C4) hemispheres (Fig. 3E). An occipital ERS was also present during this vibration burst ready period. The sustained tactile stimulation revealed a contralateral somatosensory activation; while different SS tasks resulted in distinctive cortical activation distributions, with contralateral stronger during both SS-L and SS-R task (Fig. 3A, B). In comparison, during the SAO tasks, the induced cortical activation was similar to the SS tasks (Fig. 3C, D). Moreover, occipital suppression (ERS) was shown in both real and imagined sensation tasks, with more pronounced ERS in both SAO-L and SAO-R tasks than that in SS-L and SS-R tasks. Interestingly, the frontal cortex was activated during all tasks, but no topographical difference between left and right tasks was found.

\section{B. Real-time BCI performance Calibrated with Tactile Sensation}

Fig. 4 shows the real-time BCI performance across all subjects, reaching an average accuracy of $78.8 \pm 13.1 \%$, with 12 subjects exceeding $70 \%$, and 4 subjects exceeding 90\%

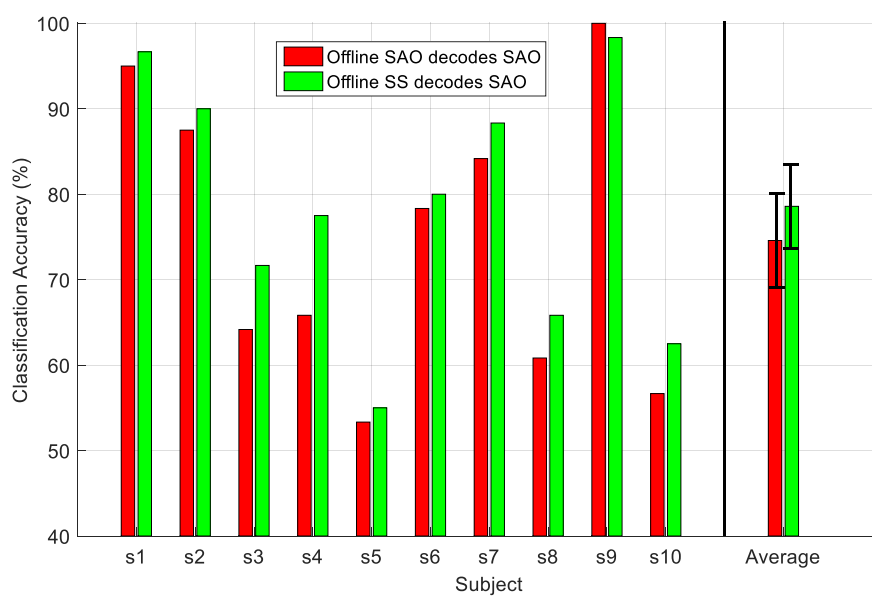

Figure 6. Offline SAO BCI performance comparison with different calibration strategy. Red bar indicates the offline performance of SAO in the last three runs when SAO in the first two runs was used for calibration; green bar indicates the offline performance of SAO in the last three runs when BCI was calibrated SS-calibration data. 
accuracy. Fig. 5 demonstrates the $\mathrm{R}^{2}$ value distribution between the left and right tasks during the real and the imagined tactile sensation. It can be observed that the discriminant feature was mostly concentrated in both the left and right sensorimotor region, and similarity existed between the SS and SAO.

\section{Offline Comparison between Different Calibrations}

The SAO in the last three runs were utilized as the common testing data set. In the conventional calibration, the SAO in the first two runs was utilized to calibrate the BCI system. In the proposed calibration, the SS data were utilized to calibrate the BCI system. Both calibrated systems were evaluated on the common SAO data set for testing. Fig. 6 illustrates the corresponding BCI decoding performance. Paired t-test showed that the proposed calibration strategy led to greater accuracy than the conventional strategy $(78.6 \pm 14.8 \%$ vs $74.6 \pm 16.6 \% ; \mathrm{p}<0.01)$.

\section{DISCUSSION}

In this study, the feasibility of a novel calibration strategy for covert somatosensory attention decoding was validated through online BCI experiments. To the best of our knowledge, this is the first time that stimulus-induced oscillatory dynamics from real sensation was utilized to facilitate the decoding of imagined sensation. The averaged online BCI performance was approximately $79 \%$, which was greater than in our recent online SAO proof-of-concept study, in which SAO data were used for both training and testing [15]. In this previous study, an SAO group of 18 subjects reached an average accuracy of $\sim 75 \%$. For within-subject offline calibration comparison, we demonstrated that the proposed calibration method was superior to the conventional method. This is somehow counter-intuitive. One would argue that the signal consistency between training and testing data in the conventional strategy is better than that of the proposed approach, because the training data and testing data were obtained with SAO in the conventional strategy, while training data and testing data were obtained with SS and SAO, respectively in the proposed approach. However, based on our results, we confirmed the current calibration approach because $\mathrm{SAO}$ is a pure mental process, which is subjective and cannot be checked directly by the experimenter. On the other hand, passive tactile sensation can be precisely administered by the experimenter. Consequently, the consistency of training data with SS is better than with SAO. In practice, this calibration procedure would be specifically useful at the beginning of the BCI training.

ERD/ERS oscillatory changes are not only correlated with real movement or imagined movement [5], [45], but also with tactile sensory processing [34]. In this study, the unilateral tactile sensation was correlated with the contralateral ERD dynamics, i.e. there was a clear band power decrease in [8 26] $\mathrm{Hz}$ alpha-beta frequency band in the contralateral somatosensory cortex (Fig. 2A). Moreover, this cortical activation was mainly concentrated in the sensorimotor cortex as shown from the topographic activation distribution in Fig. 3 (A)(B) and Fig. 5(A). By contrast, both left and right somatosensory cortices were activated when both hand wrists were stimulated by the short vibration bursts (Fig. 3E). Therefore, it was clear that the somatosensory oscillatory activation can be passively modulated by delivering stimuli to different body parts in a controllable way, and this sensationinduced activation pattern was similar to the one induced by covert somatosensory attention, as exhibited in Fig. 2(B), and the activation region was also localized on the sensorimotor region, which was in accordance with real tactile sensation, as shown in Fig. 3(C)(D) and Fig. 5(B). However, the contralateral ERD activation in real sensation was stronger than that in imagined sensation, due to the actual peripheral somatic stimulation, which presumably requires more cortical resources.

Tactile stimulus-induced oscillatory dynamics would provide a novel signal modality for tactile BCI research, and could largely improve current tactile BCI performance. The first prototype of a tactile BCI was proposed by Mueller-Putz et al [46], and based on steady-state somatosensory evoked potentials (SSSEP) [47]-[49], reaching an average accuracy of $70.4 \%$. Another similar study on SSSEP showed a mean classification accuracy of 58\% for 16 subjects [50]. Subsequently, a tactile P300 system based on the oddball paradigm, was proposed [51], [52]. This system achieved a mean accuracy of $72 \%$ in 11 subjects, when selecting between two targets. By comparison, we have shown that BCI based on tactile selective sensation has largely increased tactile BCI performance by approximately $10 \%$, and this tactile BCI modality based on SS substantially outperformed previous tactile BCI systems, making it potentially applicable to a larger number of users [36], [37]. The ERD/ERS oscillatory response and SSSEP response provide complementary information of the somatosensory input processing. Thus theoretically, in tactile BCIs, hybridizing the oscillatory dynamics and SSSEP response to tactile stimulus would provide a way to improve the tactile BCI performance.

This study further strongly supports our proof-of-concept study of SAO for a new BCI modality [15]. The ERD/ERS oscillatory changes in real and imagined sensation, as revealed from the current study, exhibited similar contralateral activation and ipsilateral suppression, as in real or imagined movement, but mainly in the somatosensory cortex. The somatosensory activation was localized around C3 and C4 EEG channels, which are also used to study MI. In our previous study [30], these oscillatory dynamics were systematically compared between the motor imagery and sensory stimulation, showing that MI and SS share a similar activation pattern and ERD/ERS dynamics in EEG, although the brain activation sources are different.

The proposed approach was partly motivated by our previous study [30], in which the concept of tendon vibration induced sensation with kinesthesia illusion was introduced to facilitate MI BCI system training. The similarity of ERD/ERS dynamics with respect to imagined movement and illusory movement lays the foundation for the proposed calibration and MI task guidance framework. The proposed stimulation assisted training paradigm, in which every illusory sensation trial was followed by a motor imagery trial, provides a way to improve MI performance in BCI setting. By contrast, in the current study, our aim was targeted at decoding the covert 
somatosensory attention (imagined sensation), which was tested online in a different BCI setting. The experimental protocol was different from our previous study: instead of the alteration between SS and SAO [30], in this study during the first two runs subjects only received real tactile sensation, while during the next three runs subjects performed imagined sensation tasks. Moreover, the stimulation devices were different, with one targeted at induced illusory movement through tendon stimulation, which needs much stronger mechanical stimulation, while the other (in the current study) was targeted at the tactile sensation level.

The proposed methodology could have potential in therapeutic applications of BCI. Specifically, for patients who suffered from an attention problem, the external stimulation may be used to passively guide the attention of the patient, after which the system can decode the covert sensory selection tasks without stimulation. Moreover, many BCI users have difficulties in complying with the instructions of the experimenter. SAO is a pure mental process without any observable objective measure, thus the therapist has no information about the compliance of the patients. The stimulation-sensation tasks are clearer to the subjects, compared to sensory imagination. In addition, many stroke patients suffer from motor impairment, while in some of these the sensory pathways might be preserved. For these patients, the current stimulation methodology together with SAO might provide a new rehabilitation approach for functional recovery. From the patient-tailored point of view, MI or SAO based BCI may be optimal depending on the cortical region of the lesion.

Considering on the experimental paradigm for the feasibility of covert somatosensory attention decoding by a BCI calibrated with tactile sensation, all subjects participated SS runs and then followed by SAO testing runs. In order to further validate the current approach, 10 (of 15) subjects were required to perform additional pre-calibration SAO runs and the performance of different calibration strategies was then compared in an offline setting. We didn't randomize the order of the pre-calibration SAO phase and the calibration SS phase in the current experimental protocol, as calibration SS phase followed by SAO (including both pre-calibration SAO and post-calibration SAO) is similar to the condition of SS phase followed by post-calibration SAO decoding runs. It is worthwhile to mention that the initial SAO runs may have facilitated the focus on the subsequent real stimulation sensation and, SAO might have been easier for the subjects after the real stimulation sensation. This training effect on SAO performance due to tactile stimulation would be worthy of future investigation, as it would provide a new way to further advance SAO performance due to sensory stimulation.

\section{CONCLUSION}

In this paper, we proposed and validated a new calibration strategy for covert somatosensory attention by delivering the tactile stimulus in a controllable way during calibration phase. This strategy exploited the fact that real and imagined sensations exhibited similarity in ERD oscillatory dynamics, and this activation was mainly concentrated on the contralateral somatosensory cortex. Our offline analysis showed that the performance was significantly better than with the conventional calibration method. And online decoding accuracy was close to $79 \%$ among 15 subjects. Due to the inherently internal nature of the SAO mental process, the stimulation assisted calibration provides a practical benefit for BCI users.

\section{ACKNOWLEDGEMENT}

We thank all volunteers for their participation in the study. This work is supported by the University Starter Grant of the University of Waterloo (No. 203859), the National Natural Science Foundation of China (Grant No. 51620105002).

\section{REFERENCE}

[1] J. R. Wolpaw et al., "Brain-computer interfaces for communication and control," Clin. Neurophysiol., vol. 113, no. 6, pp. 767-791, 2002.

[2] A. Kübler et al., "Patients with ALS can use sensorimotor rhythms to operate a brain-computer interface," Neurology, vol. 64, no. 10, pp. $1775-1777,2005$.

[3] O. Bai et al., "Towards a user-friendly brain-computer interface: initial tests in ALS and PLS patients.," Clin. Neurophysiol., vol. 121, no. 8, pp. 1293-303, Aug. 2010.

[4] M. J. Vansteensel et al., "Fully Implanted Brain-Computer Interface in a Locked-In Patient with ALS," N. Engl. J. Med., vol. 375, no. 21, p. NEJMoa1608085, 2016.

[5] G. Pfurtscheller et al., "Motor imagery activates primary sensorimotor area in humans.," Neurosci. Lett., vol. 239, no. 2-3, p. 65, 1997.

[6] C. Neuper et al., "Imagery of motor actions: differential effects of kinesthetic and visual-motor mode of imagery in single-trial EEG.," Brain Res. Cogn. Brain Res., vol. 25, no. 3, pp. 668-77, Dec. 2005.

[7] G. Pfurtscheller et al., "Mu rhythm (de)synchronization and EEG single-trial classification of different motor imagery tasks," Neuroimage, vol. 31, no. 1, pp. 153-159, 2006.

[8] G. Pfurtscheller and C. Neuper, "Motor imagery and direct braincomputer communication," Proc. IEEE, vol. 89, no. 7, pp. 11231134, 2001.

[9] B. Blankertz et al., "The BCI competition 2003: progress and perspectives in detection and discrimination of EEG single trials," IEEE Trans. Biomed. Eng., vol. 51, no. 6, pp. 1044-1051, 2004.

[10] B. Blankertz et al., "The BCI competition III: Validating alternative approaches to actual BCI problems," IEEE Trans. Neural Syst. Rehabil. Eng., vol. 14, no. 2, pp. 153-159, 2006.

[11] M. Tangermann et al., "Review of the BCI competition IV," Front. Neurosci., vol. 6, 2012.

[12] J. Meng et al., "Simultaneously optimizing spatial spectral features based on mutual information for EEG classification," IEEE Trans. Biomed. Eng., vol. 62, no. 1, pp. 227-240, 2015.

[13] F. Nijboer et al., "A P300-based brain-computer interface for people with amyotrophic lateral sclerosis.," Clin. Neurophysiol., vol. 119, no. 8, pp. 1909-16, Aug. 2008.

[14] M. Cheng et al., "Design and implementation of a brain-computer interface with high transfer rates," IEEE Trans. Biomed. Eng., vol. 49, no. 10, pp. 1181-1186, 2002.

[15] L. Yao et al., "A BCI System Based on Somatosensory Attentional Orientation," IEEE Trans. Neural Syst. Rehabil. Eng., vol. 25, no. 1, pp. 81-90, Jan. 2017.

[16] S. Whitmarsh et al., "Metacognitive awareness of covert somatosensory attention corresponds to contralateral alpha power.," Neuroimage, vol. 85 Pt 2, pp. 803-9, Jan. 2014.

[17] S. Haegens et al., "Somatosensory anticipatory alpha activity increases to suppress distracting input.," J. Cogn. Neurosci., vol. 24, no. 3, pp. 677-85, Mar. 2012.

[18] S. Haegens et al., "Top-down controlled alpha band activity in somatosensory areas determines behavioral performance in a discrimination task," J. Neurosci., vol. 31, no. 14, pp. 5197-5204, 2011.

[19] L. Yao et al., "A stimulus-independent hybrid BCI based on Motor Imagery and Somatosensory Attentional Orientation," Neural Syst. Rehabil. Eng. IEEE Trans., p. 10.1109/TNSRE.2017.2684084, 
2017.

[20] A. R. Murguialday et al., "Transition from the locked in to the completely locked-in state: a physiological analysis.," Clin. Neurophysiol., vol. 122, no. 5, pp. 925-33, May 2011.

[21] A. Kübler and N. Birbaumer, "Brain-computer interfaces and communication in paralysis: Extinction of goal directed thinking in completely paralysed patients?," Clin. Neurophysiol., vol. 119, no. 11, pp. 2658-2666, Nov. 2008.

[22] D. De Massari et al., "Brain communication in the locked-in state," Brain, vol. 136, no. 6, pp. 1989-2000, Jun. 2013.

[23] N. Birbaumer and L. G. Cohen, "Brain-computer interfaces: communication and restoration of movement in paralysis," $J$. Physiol., vol. 579, no. 3, pp. 621-636, Mar. 2007.

[24] G. R. Müller-Putz et al., "Event-related beta EEG-changes during passive and attempted foot movements in paraplegic patients," Brain Res., vol. 1137, pp. 84-91, 2007.

[25] M. Takahashi et al., "Fundamental research about electroencephalogram (EEG)-functional electrical stimulation (FES) rehabilitation system," in Rehabilitation Robotics, 2009. ICORR 2009. IEEE International Conference on, 2009, pp. 316-321.

[26] A. J. Szameitat et al., "Cortical activation during executed, imagined, observed, and passive wrist movements in healthy volunteers and stroke patients," Neuroimage, vol. 62, no. 1, pp. 266-280, 2012.

[27] V. Kaiser et al., "First steps toward a motor imagery based stroke BCI: new strategy to set up a classifier," Front. Neurosci., vol. 5, 2011.

[28] K. K. Ang et al., "A clinical study of motor imagery BCI performance in stroke by including calibration data from passive movement," in Engineering in Medicine and Biology Society $(E M B C), 2013$ 35th Annual International Conference of the IEEE, 2013, pp. 6603-6606.

[29] C. Vidaurre et al., "Neuromuscular electrical stimulation induced brain patterns to decode motor imagery.," Clin. Neurophysiol., vol. 124, no. 9, pp. 1824-34, Sep. 2013.

[30] L. Yao et al., "A novel calibration and task guidance framework for motor imagery BCI via a tendon vibration induced sensation with kinesthesia illusion.," J. Neural Eng., vol. 12, no. 1, p. 16005, Dec. 2015.

[31] G. Pfurtscheller, "Spatiotemporal ERD/ERS patterns during voluntary movement and motor imagery," Suppl. Clin. Neurophysiol., vol. 53, pp. 196-198, 2000.

[32] B. Graimann et al., "Visualization of significant ERD/ERS patterns in multichannel EEG and ECoG data," Clin. Neurophysiol., vol. 113, no. 1, pp. 43-47, 2002.

[33] N. Sharma et al., "Motor imagery: a backdoor to the motor system after stroke?," Stroke., vol. 37, no. 7, pp. 1941-52, Jul. 2006.

[34] E. Houdayer et al., "Movement preparation and cortical processing of afferent inputs in cortical tremor: an event-related (de)synchronization (ERD/ERS) study.," Clin. Neurophysiol., vol. 123, no. 6, pp. 1207-15, Jun. 2012.

[35] L. Yao et al., "Selective Sensation Based Brain-Computer Interface via Mechanical Vibrotactile Stimulation," PLoS One, vol. 8, no. 6, 2013.
[36] L. Yao et al., "Combining motor imagery with selective sensation toward a hybrid-modality BCI," IEEE Trans. Biomed. Eng., vol. 61, no. 8, pp. 2304-2312, 2014.

[37] L. Yao et al., "A multi-class tactile brain-computer interface based on stimulus-induced oscillatory dynamics," Neural Syst. Rehabil. Eng. IEEE Trans., p. 10.1109/TNSRE.2017.2731261, 2017.

[38] C. Breitwieser et al., "Stability and distribution of steady-state somatosensory evoked potentials elicited by vibro-tactile stimulation," Med. Biol. Eng. Comput., pp. 1-11, 2012.

[39] A. Delorme et al., "EEGLAB, SIFT, NFT, BCILAB, and ERICA: new tools for advanced EEG processing," Comput. Intell. Neurosci., vol. 2011, p. 10, 2011

[40] R. Oostenveld et al., "FieldTrip: open source software for advanced analysis of MEG, EEG, and invasive electrophysiological data," Comput. Intell. Neurosci., vol. 2011, p. 1, 2011.

[41] T. H. Wonnacott and R. J. Wonnacott, Introductory statistics, vol. 19690. Wiley Chichester, 1990.

[42] G. Schalk et al., "BCI2000: a general-purpose brain-computer interface (BCI) system," IEEE Trans. Biomed. Eng., vol. 51, no. 6, pp. 1034-1043, 2004.

[43] H. Ramoser et al., "Optimal spatial filtering of single trial EEG during imagined hand movement," IEEE Trans. Rehabil. Eng., vol. 8, no. 4, pp. 441-446, 2000.

[44] B. Blankertz et al., "Optimizing spatial filters for robust EEG single-trial analysis," IEEE Signal Process. Mag., vol. 25, no. 1, pp. 41-56, 2008

[45] G. Pfurtscheller and F. H. da Silva, "Event-related EEG/MEG synchronization and desynchronization: basic principles," Clin. Neurophysiol., vol. 110, no. 11, pp. 1842-1857, 1999.

[46] G. R. Müller-Putz et al., "Steady-state somatosensory evoked potentials: Suitable brain signals for brain-computer interfaces?," IEEE Trans. Neural Syst. Rehabil. Eng., vol. 14, no. 1, pp. 30-37, 2006.

[47] S. Tobimatsu et al., "Steady-state vibration somatosensory evoked potentials: physiological characteristics and tuning function," Clin. Neurophysiol., vol. 110, no. 11, pp. 1953-1958, Nov. 1999.

[48] M. Severens et al., "Transient and steady-state responses to mechanical stimulation of different fingers reveal interactions based on lateral inhibition," Clin. Neurophysiol., vol. 121, no. 12, pp. 2090-2096, 2010.

[49] C. Nangini et al., "Magnetoencephalographic study of vibrotactile evoked transient and steady-state responses in human somatosensory cortex," Neuroimage, vol. 33, no. 1, pp. 252-262, 2006.

[50] S. Ahn et al., "Achieving a hybrid brain-computer interface with tactile selective attention and motor imagery," J. Neural Eng., vol. 11, no. 6, p. 66004, Dec. 2014.

[51] A.-M. Brouwer and J. B. F. Van Erp, "A tactile P300 braincomputer interface," Front. Neurosci., vol. 4, p. 19, 2010.

[52] J. B. F. van Erp and A.-M. Brouwer, "Touch-based brain computer interfaces: state of the art," in Haptics Symposium (HAPTICS), 2014 IEEE, 2014, pp. 397-401. 